\title{
Association between body mass index (BMI) and vital capacity of college students of Zhuang nationality in China: a cross- section study
}

\author{
Peng Liu ${ }^{1, *}$, Ziliang $\mathrm{Ye}^{2, *}$, Haili Lu ${ }^{2}$, Jingjing Lu $^{2}$, Liqian Huang ${ }^{2}$, Jiangu Gong ${ }^{1}$, \\ Qiongying Deng ${ }^{1}$ and Lin $X_{u^{1}}$ \\ ${ }^{1}$ Department of Anatomy, Guangxi Medical University, Nanning, Guangxi 530021, China \\ ${ }^{2}$ Guangxi Medical University, Nanning, Guangxi 530021, China \\ *These authors have contributed equally to this work \\ Correspondence to: Peng Liu, email: drrliupeng@163.com \\ Keywords: body mass index, vital capacity, college students, Zhuang nationality, China \\ Received: June 08, $2017 \quad$ Accepted: August 07, $2017 \quad$ Published: September 08, 2017 \\ Copyright: Liu et al. This is an open-access article distributed under the terms of the Creative Commons Attribution License 3.0 \\ (CC BY 3.0), which permits unrestricted use, distribution, and reproduction in any medium, provided the original author and source \\ are credited.
}

\section{ABSTRACT}

Objective: Our study is to evaluate the association between body mass index (BMI) and vital capacity of college students of Zhuang Nationality in China.

Methods: 463 college students of Zhuang Nationality from Guangxi Medical University were selected. Basic information, body composition and vital capacity of college students were measured. According to the level of BMI, college students were divided into four groups (BMI<18.5, $18.5 \leq B M I<23.9,23.9 \leq B M I<27.9$ and $B M I \geq 27.9$ ). Multivariate logistic regression analysis was performed to assess the association between BMI and vital capacity.

Results: In male college students, there was no significant difference in vital capacity between the four groups $(3029.54 \pm 869.25,3347.06 \pm 784.54,3540.00 \pm 805.35$ and $3966.50 \pm 350.2, P=0.0727$, respectively). Multivariate regression analysis showed that after adjusting for confounding factors, no significant association was observed between $B M I$ and vital capacity $(O R=115.02,95 \% C I:-555.58 \sim 785.63 ; O R=-166.58$, 95\% CI: -1684.56 1351.41; OR=-484.01, 95\% CI:-3504.53, 2536.51, respectively. $B M I<18.5$ group served as reference group). In female college students, there was also no significant difference in vital capacity between the four groups (2455.15 $\$ 574.4$, 2555.06 $\pm 637.03,2750.33 \pm 1224.05$ and $2473.00 \pm 159.06, P=0.4011$, respectively). Multivariate regression analysis showed that after adjusting for confounding factors, no significant association was observed between $B M I$ and vital capacity $(O R=-88.88$, 95\% CI: $-333.59 \sim 155.84$; OR=20.00, 95\% CI: $-694.39 \sim 734.39$; OR=2.86, $95 \%$ CI: $-1830.58,1836.3$, respectively. BMI<18.5 group served as reference group).

Conclusion: There was no evidence that BMI is associated with vital capacity in college students of Zhuang Nationality.

\section{INTRODUCTION}

In recent years, overweight and obesity have become global epidemics, and are attributed to changes in living standards not only in developed countries but also in developing countries [1]. Research results have shown that $35 \%$ of adults aged $\geq 20$ years were reported to be overweight and $11 \%$ were reported to be obese in 2008 year [2-4]. However, obese or overweight people are growing year by year with the rapid economic 
development and changes in eating habits [5, 6]. In 2012 year, more than 40 million children aged $\geq 5$ years were reported to be overweight or obese all over the world $[7,8]$.

As is known to all, obesity is a major health issue all over the world. Obesity or/and overweight are a major risk factor for non-communicable disorders as well as cardiovascular disorders [9], such as hypertension [10], diabetes mellitus [11] and dyslipidemia [12], and is closely related to morbidity and mortality. The importance of weight control has been emphasized for the primary and secondary prevention of lifestyle-related disorders on a global scale.

The associations of obesity with reduced pulmonary function and lifestyle-related disorders have received considerable attention. In particular, a close relationship between obesity and restrictive pulmonary dysfunction has been suggested [13-15]. Obesity impacts on many areas of clinical medicine, including pulmonary medicine [16]. Nevertheless, it is debated if obesity is linked to asthma, or whether the obesity, due to its effect of decreasing lung volumes and increasing airway resistance, cause symptoms that simply mimic asthma. Therefore, it is important to understand the relationship between BMI and vital capacity. So far, several previous studies have reported that increased low body weight or BMI is related to reduction of vital capacity, and these results also suggest that the maintenance of adequate body weight may be important for improving the vital capacity $[17,18]$. However, evidence is limited with regard to whether BMI is associated with vital capacity among college students of Zhuang Nationality in China.

In our study, we aimed to examine the associations between BMI and vital capacity among college students of Zhuang Nationality in China, and provide theoretical reference for Chinese college students' physical health.

\section{RESULTS}

\section{Basic characteristics for all college students}

Table 1 shows the basic characteristics, including age, body fat, fat, muscle, presumption of bone, body moisture, protein, intracellular fluid, extracellular fluid, body fat percentage, muscle volume ratio, visceral fat area, visceral fat, fat content, waist hip ratio, basal metabolism, energy metabolism, swelling index, trunk muscle the amount of the left upper limb muscles, weight, left lower limb muscle mass, right upper limb muscle, right lower limb muscle mass, trunk fat, trunk fat ratio, left upper limb fat, the left upper limb left lower limb fat, left leg fat, right upper limb fat volume, right upper extremity fat percentage and right lower extremity fat mass, for all college students grouping by sex (121 male college students and 342 female college students).

\section{Basic characteristics for male college students grouping by BMI}

Table 2 shows the basic characteristics, including age, body fat, fat, muscle, presumption of bone, body moisture, protein, intracellular fluid, extracellular fluid, body fat percentage, muscle volume ratio, visceral fat area, visceral fat, fat content, waist hip ratio, basal metabolism, energy metabolism, swelling index, trunk muscle the amount of the left upper limb muscles, weight, left lower limb muscle mass, right upper limb muscle, right lower limb muscle mass, trunk fat, trunk fat ratio, left upper limb fat, the left upper limb left lower limb fat, left leg fat, right upper limb fat volume, right upper extremity fat percentage and right lower extremity fat mass, for male college students grouping by BMI. From Table 2, we can see that the basic characteristics of four groups of male college students has statistical difference except for age $(\mathrm{P}<0.05)$.

\section{Basic characteristics for female college students grouping by BMI}

Table 3 shows the basic characteristics, including age, body fat, fat, muscle, presumption of bone, body moisture, protein, intracellular fluid, extracellular fluid, body fat percentage, muscle volume ratio, visceral fat area, visceral fat, fat content, waist hip ratio, basal metabolism, energy metabolism, swelling index, trunk muscle the amount of the left upper limb muscles, weight, left lower limb muscle mass, right upper limb muscle, right lower limb muscle mass, trunk fat, trunk fat ratio, left upper limb fat, the left upper limb left lower limb fat, left leg fat, right upper limb fat volume, right upper extremity fat percentage and right lower extremity fat mass, for male college students grouping by BMI. From Table 3, we can see that the basic characteristics of four groups of female college students has statistical difference except for age $(\mathrm{P}<0.05)$.

\section{Multiple regression analysis}

Table 4 shows the association between BMI and VC of college students. In male college students, there was no significant difference in vital capacity between the four groups $(3029.54 \pm 869.25,3347.06 \pm 784.54$, $3540.00 \pm 805.35$ and $3966.50 \pm 350.2, \quad \mathrm{P}=0.0727$, respectively) (Figure 1). Body composition, such as body fat, fat, muscle, presumption of bone, body moisture, protein and intracellular fluid, may have an effect on lung capacity. Therefore, multivariate regression analysis was performed to adjust confounding factors. Multivariate regression analysis showed that after adjusting for confounding factors, no significant association was observed between BMI and vital capacity 
Table 1: Baseline clinical characteristics

\begin{tabular}{|c|c|c|}
\hline Gender & Male & Female \\
\hline $\mathrm{N}$ & 121 & 342 \\
\hline Height (cm) & $167.95 \pm 5.18$ & $156.87 \pm 4.75$ \\
\hline Age (year) & $19.75 \pm 1.44$ & $19.97 \pm 1.37$ \\
\hline Weight (kg) & $58.23 \pm 9.38$ & $47.64 \pm 5.84$ \\
\hline BMI $\left(\mathrm{kg} / \mathrm{m}^{2}\right)$ & $20.58 \pm 2.79$ & $19.34 \pm 2.21$ \\
\hline Lean body weight $(\mathrm{kg})$ & $50.46 \pm 5.41$ & $36.39 \pm 2.86$ \\
\hline Fat content $(\mathrm{kg})$ & $7.78 \pm 5.06$ & $11.27 \pm 3.91$ \\
\hline Muscle content $(\mathrm{kg})$ & $47.83 \pm 5.13$ & $34.37 \pm 2.61$ \\
\hline Presumptive bone mass $(\mathrm{kg})$ & $2.63 \pm 0.28$ & $2.02 \pm 0.25$ \\
\hline Body moisture (kg) & $35.04 \pm 4.27$ & $25.44 \pm 2.27$ \\
\hline Protein $(\mathrm{kg})$ & $12.84 \pm 2.08$ & $8.97 \pm 0.87$ \\
\hline Intracellular fluid $(\mathrm{kg})$ & $21.91 \pm 3.14$ & $15.78 \pm 1.48$ \\
\hline Extracellular fluid (kg) & $13.16 \pm 1.23$ & $9.69 \pm 0.90$ \\
\hline Body fat percentage (\%) & $12.58 \pm 5.91$ & $23.12 \pm 5.30$ \\
\hline Muscle volume ratio (\%) & $94.99 \pm 9.16$ & $95.00 \pm 6.22$ \\
\hline Visceral fat area $\left(\mathrm{cm}^{2}\right)$ & $31.08 \pm 26.87$ & $13.21 \pm 8.81$ \\
\hline Visceral fat content $(\mathrm{kg})$ & $0.86 \pm 0.97$ & $0.78 \pm 0.51$ \\
\hline Subcutaneous fat content $(\mathrm{kg})$ & $6.88 \pm 4.12$ & $10.49 \pm 3.44$ \\
\hline Waist to hip ratio (\%) & $0.86 \pm 0.04$ & $0.78 \pm 0.03$ \\
\hline Basal metabolism (kcal/d) & $1460.08 \pm 164.18$ & $1130.53 \pm 89.32$ \\
\hline Total energy metabolism ( $\mathrm{kcal} / \mathrm{d})$ & $2168.21 \pm 243.82$ & $1678.86 \pm 132.64$ \\
\hline Edema index (\%) & $0.36 \pm 0.01$ & $0.36 \pm 0.01$ \\
\hline Trunk muscle mass (kg) & $23.99 \pm 2.58$ & $17.45 \pm 1.68$ \\
\hline Left upper limb muscle mass (kg) & $2.46 \pm 0.33$ & $1.57 \pm 0.67$ \\
\hline Left lower limb muscle mass (kg) & $9.37 \pm 1.17$ & $6.91 \pm 0.56$ \\
\hline Right upper limb muscle mass ( $\mathrm{kg}$ ) & $2.54 \pm 0.30$ & $1.55 \pm 0.18$ \\
\hline Right lower limb muscle mass $(\mathrm{kg})$ & $9.57 \pm 1.17$ & $7.00 \pm 0.51$ \\
\hline Trunk fat mass $(\mathrm{kg})$ & $4.10 \pm 3.13$ & $5.00 \pm 2.32$ \\
\hline Trunk fat rate $(\%)$ & $12.92 \pm 7.37$ & $20.56 \pm 6.87$ \\
\hline Left upper limb fat mass (kg) & $0.33 \pm 0.17$ & $0.46 \pm 0.27$ \\
\hline Left upper extremity fat percentage (\%) & $10.08 \pm 4.56$ & $20.17 \pm 5.90$ \\
\hline Left lower limb fat mass $(\mathrm{kg})$ & $1.57 \pm 0.81$ & $2.73 \pm 0.63$ \\
\hline Left lower extremity fat rate (\%) & $12.93 \pm 4.68$ & $26.78 \pm 3.89$ \\
\hline Right upper limb fat mass (kg) & $0.31 \pm 0.17$ & $0.42 \pm 0.19$ \\
\hline Right upper limb fat rate $(\%)$ & $9.36 \pm 4.39$ & $18.67 \pm 5.72$ \\
\hline Right lower extremity fat mass $(\mathrm{kg})$ & $1.58 \pm 0.83$ & $2.77 \pm 0.63$ \\
\hline Right lower extremity fat rate (\%) & $12.78 \pm 4.67$ & $26.77 \pm 3.73$ \\
\hline
\end{tabular}

Data are presented as mean $\pm \mathrm{SD}$. 
Table 2: Basic characteristics for male college students grouping by BMI

\begin{tabular}{|c|c|c|c|c|c|}
\hline BMI & $<18.5$ & $>=18.5,<23.9$ & $>=23.9,<27.9$ & $>=27.9$ & P-value \\
\hline $\mathrm{N}$ & 28 & 79 & 10 & 4 & \\
\hline Age (year) & $19.57 \pm 1.26$ & $19.82 \pm 1.53$ & $19.80 \pm 1.40$ & $19.50 \pm 1.29$ & 0.862 \\
\hline Lean body weight (kg) & $45.77 \pm 2.63$ & $50.77 \pm 4.26$ & $56.05 \pm 5.37$ & $63.15 \pm 4.65$ & $<0.001$ \\
\hline Fat content $(\mathrm{kg})$ & $4.16 \pm 1.70$ & $7.19 \pm 3.04$ & $16.20 \pm 3.16$ & $23.77 \pm 4.19$ & $<0.001$ \\
\hline Muscle content (kg) & $43.37 \pm 2.50$ & $48.13 \pm 4.05$ & $53.15 \pm 5.08$ & $59.88 \pm 4.42$ & $<0.001$ \\
\hline Presumptive bone mass (kg) & $2.40 \pm 0.14$ & $2.65 \pm 0.22$ & $2.90 \pm 0.29$ & $3.27 \pm 0.24$ & $<0.001$ \\
\hline Body moisture (kg) & $31.52 \pm 2.53$ & $35.59 \pm 3.75$ & $37.99 \pm 4.83$ & $41.30 \pm 5.49$ & $<0.001$ \\
\hline Protein $(\mathrm{kg})$ & $11.89 \pm 0.99$ & $12.58 \pm 1.73$ & $15.19 \pm 1.89$ & $18.60 \pm 1.63$ & $<0.001$ \\
\hline Intracellular fluid $(\mathrm{kg})$ & $19.53 \pm 2.01$ & $22.38 \pm 2.90$ & $23.51 \pm 3.64$ & $25.20 \pm 4.13$ & $<0.001$ \\
\hline Extracellular fluid (kg) & $12.02 \pm 0.54$ & $13.24 \pm 0.90$ & $14.52 \pm 1.22$ & $16.12 \pm 1.41$ & $<0.001$ \\
\hline Body fat percentage (\%) & $8.26 \pm 3.32$ & $12.14 \pm 4.25$ & $22.24 \pm 2.96$ & $27.35 \pm 4.83$ & $<0.001$ \\
\hline Muscle volume ratio (\%) & $86.67 \pm 3.79$ & $95.35 \pm 6.77$ & $106.70 \pm 8.30$ & $116.75 \pm 9.73$ & $<0.001$ \\
\hline Visceral fat area $\left(\mathrm{cm}^{2}\right)$ & $10.00 \pm 0.00$ & $28.09 \pm 15.29$ & $77.90 \pm 10.79$ & $119.85 \pm 9.77$ & $<0.001$ \\
\hline Visceral fat content $(\mathrm{kg})$ & $0.29 \pm 0.17$ & $0.69 \pm 0.48$ & $2.43 \pm 0.62$ & $4.42 \pm 0.96$ & $<0.001$ \\
\hline Subcutaneous fat content (kg) & $3.85 \pm 1.54$ & $6.45 \pm 2.56$ & $13.75 \pm 2.54$ & $19.35 \pm 3.28$ & $<0.001$ \\
\hline Waist to hip ratio $(\%)$ & $0.83 \pm 0.03$ & $0.86 \pm 0.03$ & $0.93 \pm 0.01$ & $0.98 \pm 0.02$ & $<0.001$ \\
\hline Basal metabolism (kcal/d) & $1318.57 \pm 71.22$ & $1466.28 \pm 124.31$ & $1641.40 \pm 169.38$ & $1875.00 \pm 132.93$ & $<0.001$ \\
\hline Total energy metabolism (kcal/d) & $1958.04 \pm 105.78$ & $2177.42 \pm 184.61$ & $2437.60 \pm 251.44$ & $2784.25 \pm 197.20$ & $<0.001$ \\
\hline Edema index $(\%)$ & $0.36 \pm 0.01$ & $0.35 \pm 0.01$ & $0.36 \pm 0.01$ & $0.36 \pm 0.01$ & 0.044 \\
\hline Trunk muscle mass (kg) & $21.94 \pm 1.50$ & $24.13 \pm 2.08$ & $26.21 \pm 2.60$ & $29.93 \pm 3.27$ & $<0.001$ \\
\hline Left upper limb muscle mass (kg) & $2.22 \pm 0.19$ & $2.50 \pm 0.32$ & $2.65 \pm 0.35$ & $2.77 \pm 0.26$ & $<0.001$ \\
\hline Left lower limb muscle mass (kg) & $8.43 \pm 0.54$ & $9.41 \pm 0.97$ & $10.64 \pm 1.13$ & $12.08 \pm 0.68$ & $<0.001$ \\
\hline Right upper limb muscle mass (kg) & $2.31 \pm 0.21$ & $2.58 \pm 0.26$ & $2.73 \pm 0.35$ & $2.95 \pm 0.26$ & $<0.001$ \\
\hline Right lower extremity muscle mass (kg) & $8.58 \pm 0.51$ & $9.61 \pm 0.92$ & $11.01 \pm 1.21$ & $12.28 \pm 0.70$ & $<0.001$ \\
\hline Trunk fat mass $(\mathrm{kg})$ & $2.07 \pm 1.17$ & $3.66 \pm 1.94$ & $9.11 \pm 2.01$ & $14.22 \pm 2.47$ & $<0.001$ \\
\hline Trunk fat rate $(\%)$ & $8.08 \pm 4.38$ & $12.23 \pm 5.49$ & $24.68 \pm 3.96$ & $31.20 \pm 5.55$ & $<0.001$ \\
\hline Left upper limb fat mass (kg) & $0.20 \pm 0.07$ & $0.32 \pm 0.11$ & $0.62 \pm 0.14$ & $0.75 \pm 0.17$ & $<0.001$ \\
\hline Left upper extremity fat percentage $(\%)$ & $6.56 \pm 2.36$ & $9.83 \pm 3.26$ & $17.82 \pm 2.45$ & $20.30 \pm 5.35$ & $<0.001$ \\
\hline Left lower limb fat mass (kg) & $0.92 \pm 0.28$ & $1.51 \pm 0.48$ & $2.91 \pm 0.55$ & $4.00 \pm 0.87$ & $<0.001$ \\
\hline Left lower extremity fat rate $(\%)$ & $9.17 \pm 2.93$ & $12.79 \pm 3.37$ & $20.33 \pm 2.13$ & $23.70 \pm 4.50$ & $<0.001$ \\
\hline Right upper limb fat mass (kg) & $0.18 \pm 0.07$ & $0.30 \pm 0.11$ & $0.60 \pm 0.12$ & $0.78 \pm 0.21$ & $<0.001$ \\
\hline Right upper limb fat rate $(\%)$ & $5.99 \pm 2.23$ & $9.11 \pm 3.17$ & $16.88 \pm 2.25$ & $19.20 \pm 5.02$ & $<0.001$ \\
\hline Right lower extremity fat mass (kg) & $0.90 \pm 0.27$ & $1.52 \pm 0.47$ & $3.00 \pm 0.56$ & $4.12 \pm 0.75$ & $<0.001$ \\
\hline Right lower extremity fat rate (\%) & $8.86 \pm 2.81$ & $12.64 \pm 3.22$ & $20.30 \pm 2.31$ & $24.00 \pm 3.96$ & $<0.001$ \\
\hline
\end{tabular}

Data are presented as mean $\pm \mathrm{SD}$. 
Table 3: Basic characteristics for female college students grouping by BMI

\begin{tabular}{|c|c|c|c|c|c|}
\hline BMI & $<18.5$ & $>=18.5,<23.9$ & $>=23.9,<27.9$ & $>=27.9$ & P-value \\
\hline $\mathrm{N}$ & 109 & 221 & 9 & 3 & \\
\hline Age (year) & $20.12 \pm 1.41$ & $19.91 \pm 1.35$ & $19.56 \pm 1.42$ & $20.33 \pm 0.58$ & 0.442 \\
\hline Lean body weight (kg) & $34.71 \pm 2.25$ & $37.01 \pm 2.66$ & $40.28 \pm 3.64$ & $40.17 \pm 1.02$ & $<0.001$ \\
\hline Fat content $(\mathrm{kg})$ & $7.94 \pm 1.90$ & $12.24 \pm 2.43$ & $21.78 \pm 2.31$ & $29.90 \pm 3.65$ & $<0.001$ \\
\hline Muscle content (kg) & $32.83 \pm 2.06$ & $34.93 \pm 2.43$ & $37.91 \pm 3.33$ & $37.80 \pm 0.96$ & $<0.001$ \\
\hline Presumptive bone mass (kg) & $1.88 \pm 0.20$ & $2.08 \pm 0.24$ & $2.37 \pm 0.31$ & $2.33 \pm 0.06$ & $<0.001$ \\
\hline Body moisture (kg) & $23.85 \pm 1.55$ & $25.97 \pm 1.96$ & $29.21 \pm 2.50$ & $32.13 \pm 0.81$ & $<0.001$ \\
\hline Protein (kg) & $9.02 \pm 0.70$ & $9.00 \pm 0.86$ & $8.72 \pm 1.10$ & $5.73 \pm 0.12$ & $<0.001$ \\
\hline Intracellular fluid (kg) & $14.94 \pm 1.09$ & $16.07 \pm 1.40$ & $17.41 \pm 1.69$ & $19.23 \pm 0.99$ & $<0.001$ \\
\hline Extracellular fluid (kg) & $8.94 \pm 0.54$ & $9.93 \pm 0.66$ & $11.81 \pm 0.92$ & $12.93 \pm 0.29$ & $<0.001$ \\
\hline Body fat percentage $(\%)$ & $18.42 \pm 3.52$ & $24.69 \pm 3.56$ & $35.11 \pm 2.29$ & $42.60 \pm 3.44$ & $<0.001$ \\
\hline Muscle volume ratio (\%) & $91.04 \pm 4.77$ & $96.37 \pm 5.30$ & $102.41 \pm 8.73$ & $116.10 \pm 2.04$ & $<0.001$ \\
\hline Visceral fat area $\left(\mathrm{cm}^{2}\right)$ & $10.09 \pm 0.96$ & $12.81 \pm 5.93$ & $43.78 \pm 10.31$ & $64.17 \pm 13.16$ & $<0.001$ \\
\hline Visceral fat content $(\mathrm{kg})$ & $0.45 \pm 0.18$ & $0.85 \pm 0.30$ & $2.37 \pm 0.54$ & $3.67 \pm 0.91$ & $<0.001$ \\
\hline Subcutaneous fat content $(\mathrm{kg})$ & $7.48 \pm 1.74$ & $11.41 \pm 2.14$ & $19.42 \pm 1.77$ & $26.20 \pm 2.74$ & $<0.001$ \\
\hline Waist to hip ratio $(\%)$ & $0.75 \pm 0.02$ & $0.79 \pm 0.02$ & $0.84 \pm 0.02$ & $0.87 \pm 0.02$ & $<0.001$ \\
\hline Basal metabolism (kcal/.d) & $1066.97 \pm 62.99$ & $1152.64 \pm 77.18$ & $1292.78 \pm 101.56$ & $1324.33 \pm 24.83$ & $<0.001$ \\
\hline Total energy metabolism (kcal/.d) & $1584.46 \pm 93.52$ & $1711.71 \pm 114.62$ & $1919.67 \pm 150.89$ & $1966.33 \pm 36.95$ & $<0.001$ \\
\hline Edema index $(\%)$ & $0.35 \pm 0.01$ & $0.36 \pm 0.01$ & $0.37 \pm 0.01$ & $0.37 \pm 0.01$ & $<0.001$ \\
\hline Trunk muscle mass $(\mathrm{kg})$ & $16.68 \pm 1.39$ & $17.77 \pm 1.66$ & $19.06 \pm 2.18$ & $17.27 \pm 0.38$ & $<0.001$ \\
\hline Left upper limb muscle mass (kg) & $1.46 \pm 0.60$ & $1.61 \pm 0.71$ & $1.76 \pm 0.17$ & $2.00 \pm 0.00$ & 0.127 \\
\hline Left lower limb muscle mass (kg) & $6.65 \pm 0.48$ & $6.99 \pm 0.53$ & $7.63 \pm 0.56$ & $8.23 \pm 0.51$ & $<0.001$ \\
\hline Right upper limb muscle mass (kg) & $1.42 \pm 0.13$ & $1.59 \pm 0.15$ & $1.81 \pm 0.23$ & $2.07 \pm 0.06$ & $<0.001$ \\
\hline Right lower extremity muscle mass (kg) & $6.72 \pm 0.40$ & $7.09 \pm 0.47$ & $7.74 \pm 0.50$ & $8.30 \pm 0.40$ & $<0.001$ \\
\hline Trunk fat mass $(\mathrm{kg})$ & $3.10 \pm 1.18$ & $5.54 \pm 1.53$ & $11.41 \pm 1.73$ & $15.10 \pm 2.26$ & $<0.001$ \\
\hline Trunk fat rate $(\%)$ & $14.62 \pm 4.80$ & $22.52 \pm 4.69$ & $36.02 \pm 3.19$ & $45.13 \pm 4.27$ & $<0.001$ \\
\hline Left upper limb fat mass (kg) & $0.31 \pm 0.18$ & $0.50 \pm 0.24$ & $0.94 \pm 0.12$ & $1.60 \pm 0.17$ & $<0.001$ \\
\hline Left upper extremity fat percentage $(\%)$ & $15.22 \pm 4.08$ & $21.78 \pm 4.13$ & $33.28 \pm 2.35$ & $42.17 \pm 3.59$ & $<0.001$ \\
\hline Left lower limb fat mass $(\mathrm{kg})$ & $2.17 \pm 0.32$ & $2.90 \pm 0.37$ & $4.29 \pm 0.28$ & $5.83 \pm 0.55$ & $<0.001$ \\
\hline Left lower extremity fat rate $(\%)$ & $23.34 \pm 2.86$ & $27.98 \pm 2.68$ & $34.56 \pm 1.31$ & $39.97 \pm 2.35$ & $<0.001$ \\
\hline Right upper limb fat mass (kg) & $0.27 \pm 0.08$ & $0.46 \pm 0.11$ & $0.90 \pm 0.10$ & $1.57 \pm 0.15$ & $<0.001$ \\
\hline Right upper limb fat rate $(\%)$ & $13.56 \pm 3.64$ & $20.35 \pm 3.77$ & $31.73 \pm 2.59$ & $41.20 \pm 3.32$ & $<0.001$ \\
\hline Right lower extremity fat mass (kg) & $2.21 \pm 0.32$ & $2.94 \pm 0.37$ & $4.34 \pm 0.28$ & $5.90 \pm 0.52$ & $<0.001$ \\
\hline Right lower extremity fat rate $(\%)$ & $23.44 \pm 2.65$ & $27.93 \pm 2.52$ & $34.44 \pm 1.20$ & $39.87 \pm 2.50$ & $<0.001$ \\
\hline
\end{tabular}

Data are presented as mean $\pm \mathrm{SD}$. 
Table 4: Multiple regression analysis

\begin{tabular}{|c|c|c|}
\hline Exposure & Male & Female \\
\hline \multicolumn{3}{|l|}{ Non-adjusted } \\
\hline \multicolumn{3}{|l|}{ BMI } \\
\hline$<18.5$ & ref & ref \\
\hline$>=18.5,<23.9$ & $317.53(-26.72,661.78) 0.0732$ & $99.91(-46.11,245.94) 0.1808$ \\
\hline$>=23.9,<27.9$ & $510.46(-66.16,1087.09) 0.0854$ & $295.19(-137.51,727.88) 0.1821$ \\
\hline$>=27.9$ & $936.96(100.31,1773.61) 0.0301$ & $17.85(-712.29,748.00) 0.9618$ \\
\hline \multicolumn{3}{|l|}{ Adjust I } \\
\hline \multicolumn{3}{|l|}{ BMI } \\
\hline$<18.5$ & ref & ref \\
\hline$>=18.5,<23.9$ & $291.47(-48.9,631.84) 0.0960$ & $106.19(-40.09,252.46) 0.1557$ \\
\hline$>=23.9,<27.9$ & $486.77(-82.29,1055.83) 0.0963$ & $312.42(-120.88,745.72) 0.1585$ \\
\hline$>=27.9$ & $944.37(119.29,1769.44) 0.0268$ & $11.31(-718.41,741.03) 0.9758$ \\
\hline \multicolumn{3}{|l|}{ Adjust II } \\
\hline \multicolumn{3}{|l|}{ BMI } \\
\hline$<18.5$ & ref & ref \\
\hline$>=18.5,<23.9$ & $115.02(-555.58,785.63) 0.7376$ & $-88.88(-333.59,155.84) 0.4771$ \\
\hline$>=23.9,<27.9$ & $-166.58(-1684.56,1351.41) 0.8302$ & $20.00(-694.39,734.39) 0.9563$ \\
\hline$>=27.9$ & $-484.01(-3504.53,2536.51) 0.7543$ & $2.86(-1830.58,1836.3) 0.9976$ \\
\hline
\end{tabular}

Results are presented as OR (95\% CI) P value.

Non-adjusted model adjust for: none.

Adjust I model adjust for: age.

Adjust II model adjust for: age; body fat; fat; muscle; presumption of bone; body moisture; protein; intracellular fluid; extracellular fluid; body fat percentage; muscle volume ratio; visceral fat area; visceral fat; fat content; waist hip ratio; basal metabolism; energy metabolism; swelling index; trunk muscle the amount of the left upper limb muscles; weight; left lower limb muscle mass; right upper limb muscle; right lower limb muscle mass; trunk fat; trunk fat ratio; left upper limb fat; the left upper limb left lower limb fat; fat; left leg fat; right upper limb fat Volume; right upper extremity fat percentage; right lower extremity fat mass.

$(\mathrm{OR}=115.02 ， 95 \%$ CI: $-555.58 \sim 785.63 ; \mathrm{OR}=-166.58$, 95\% CI: $-1684.56 \sim 1351.41$; OR $=-484.01,95 \%$ CI:3504.53 , 2536.51, respectively. BMI $<18.5$ group served as reference group).

In female college students, there was also no significant difference in vital capacity between the four groups $(2455.15 \pm 574.4,2555.06 \pm 637.03$, $2750.33 \pm 1224.05$ and $2473.00 \pm 159.06, \quad \mathrm{P}=0.4011$, respectively) (Figure 2). Body composition, such as body fat, fat, muscle, presumption of bone, body moisture, protein and intracellular fluid, may have an effect on lung capacity. Therefore, multivariate regression analysis was performed to adjust confounding factors. Multivariate regression analysis showed that after adjusting for confounding factors, no significant association was observed between BMI and vital capacity $(\mathrm{OR}=-$
88.88, 95\% CI: $-333.59 \sim 155.84$; OR=20.00, 95\% CI: -694.39 734.39; OR=2.86, 95\% CI: $-1830.58,1836.3$, respectively. BMI $<18.5$ group served as reference group).

\section{DISCUSSION}

In our study, we carried out a cross-sectional study to evaluate the association between BMI and VC of college students of Zhuang Nationality in China. 463 college students were recruited in this study, and multivariate logistic regression analysis was performed to adjust confounding factors. Our result found that there was no significant difference in vital capacity between the four groups (underweight, normal BMI, overweight and obese) in male and female college students (all $\mathrm{P}>0.05$ ). In addition, after multivariate regression analysis was 
conducted, no significant association was observed between BMI and VC in male and female college students. Therefore, the results from our study suggest that there was no evidence that BMI is associated with VC in college students of Zhuang Nationality in china.

Breathing is an essential function in lung function, which can hinder quality of life and performance in activities of daily living. To maintain respiratory homeostasis, the structures that compose the respiratory system need to work in equilibrium. It means that the lungs should be ventilated and the gases should diffuse through the alveolar-capillary barrier. So far, the efficient method to estimate lung function of college student is by determining $\mathrm{VC}$, which offer information essential for the characterization of the pathophysiological state resulting from abnormalities in the pulmonary-ventilatory processes [19]. Research findings have pointed out that there are many factors that can affect VC, such as weight

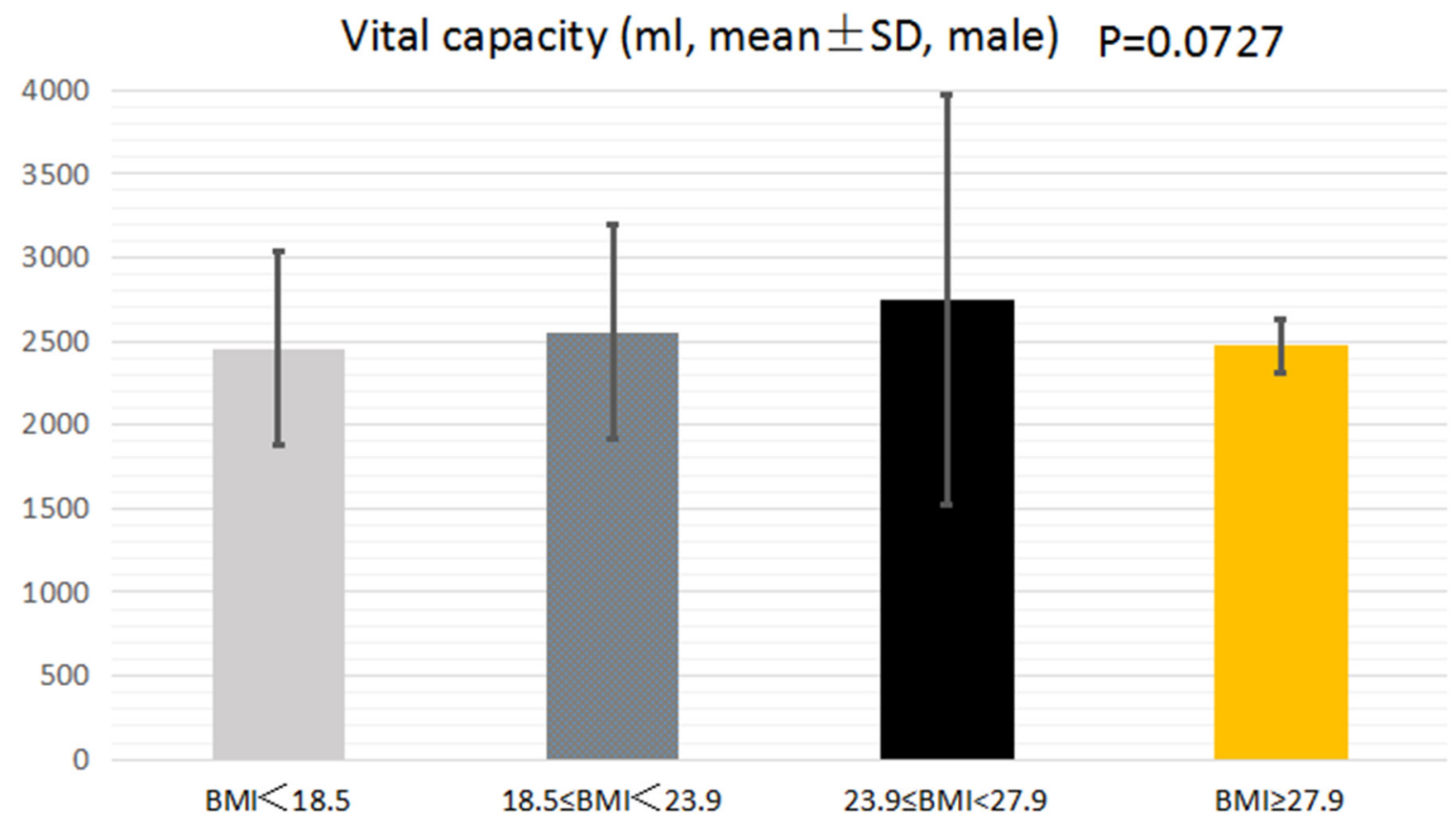

Figure 1: The association between BMI and VC of male college students.

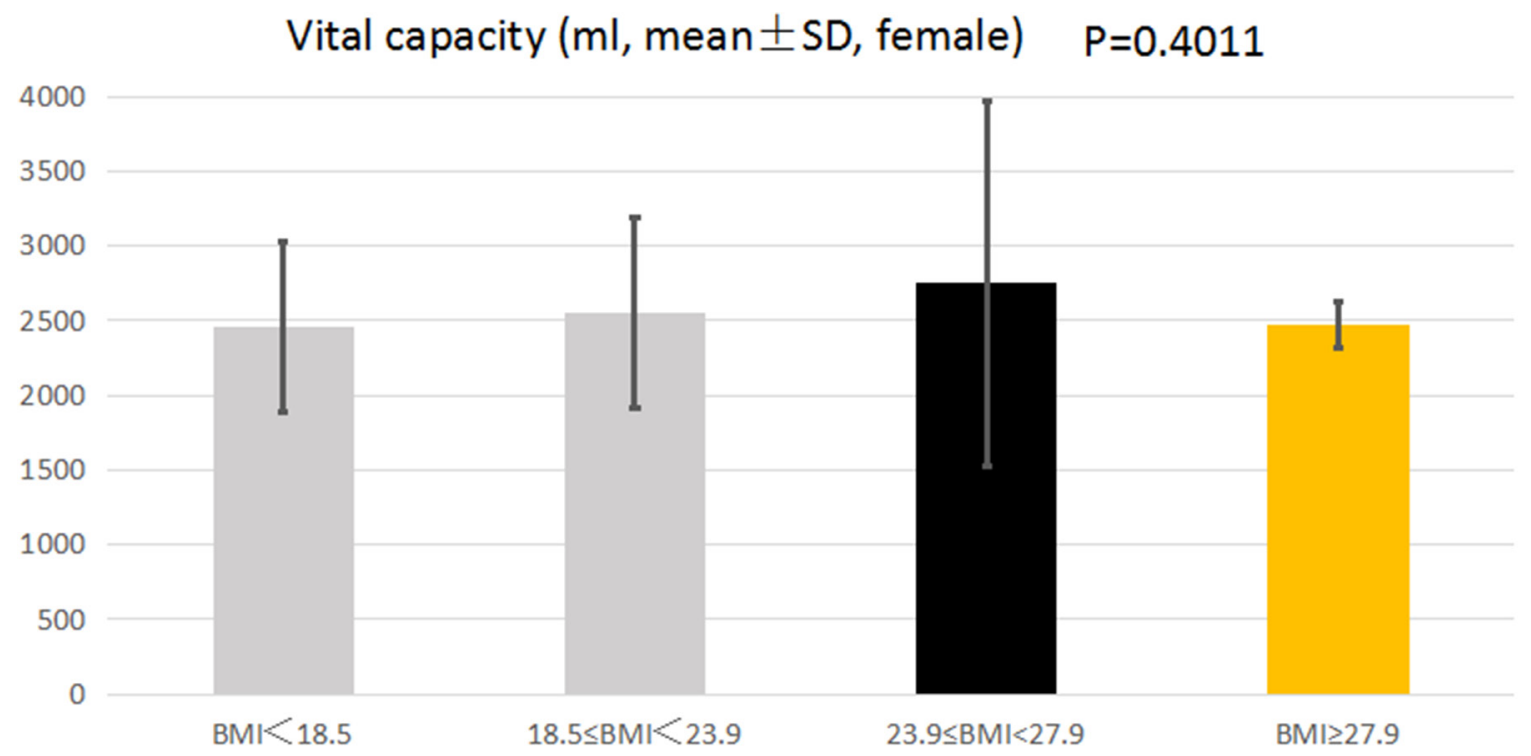

Figure 2: The association between BMI and VC of female college students. 
[20], bone mineral density [21], chronic obstructive pulmonary disease [22], BMI [17], hypertension [23] and diabetes [24]. However, few studies have been done on body mass index and vital capacity, especially in college students. Fukahori S and his colleague [25] conducted a study to evaluate the association between BMI and forced expiratory volume in 1 second/forced vital capacity in a population with a relatively low prevalence of obesity, and 1231 patients were recruited in this research. The result of this study found that BMI was positively correlated with forced expiratory volume in 1 second (FEV (1))/ forced vital capacity (FVC) in men and with maximum mid-expiratory flow (MMF) in all subjects. After adjusting for related factors using multiple regression analysis, a significant positive correlation between BMI and FEV (1)/ FVC was identified for all subjects. Finally, the authors pointed out that high BMI may be inappropriate as a predictor of obstructive lung dysfunction, particularly in populations with a low prevalence of obesity. In 2014 year, Melo LC and his colleague [26] carried out a systematic review to assess whether obesity is associated with lung function. Nine studies were selected by selecting publications in the science databases MEDLINE and LILACS. The result showed that the obese individuals presented with a reduction in lung volume and capacity as compared to healthy individuals, which means that the presence of a restrictive respiratory pattern associated with obesity. The results of these studies are difference from our results.

At present, there are several potential mechanisms by which BMI might lead to reduce $\mathrm{VC}$, which was broadly divided into mechanical and inflammatory [27]. As BMI continues to rise, the fat content rises gradually, Intra-peritoneal fat deposits and accumulation may impede the descent of the diaphragm during inspiration, which would affect the lung's breathing function [28]. In addition, an increase in abdominal fat volume can reduce the expiratory reserve volume, by displacing the diaphragm upward and reducing functional volume in the thoracic cavity [29]. Furthermore, the deposition of fat on the chest wall have negative effects on the expansion and excursion of the rib cage, through a direct loading effect or

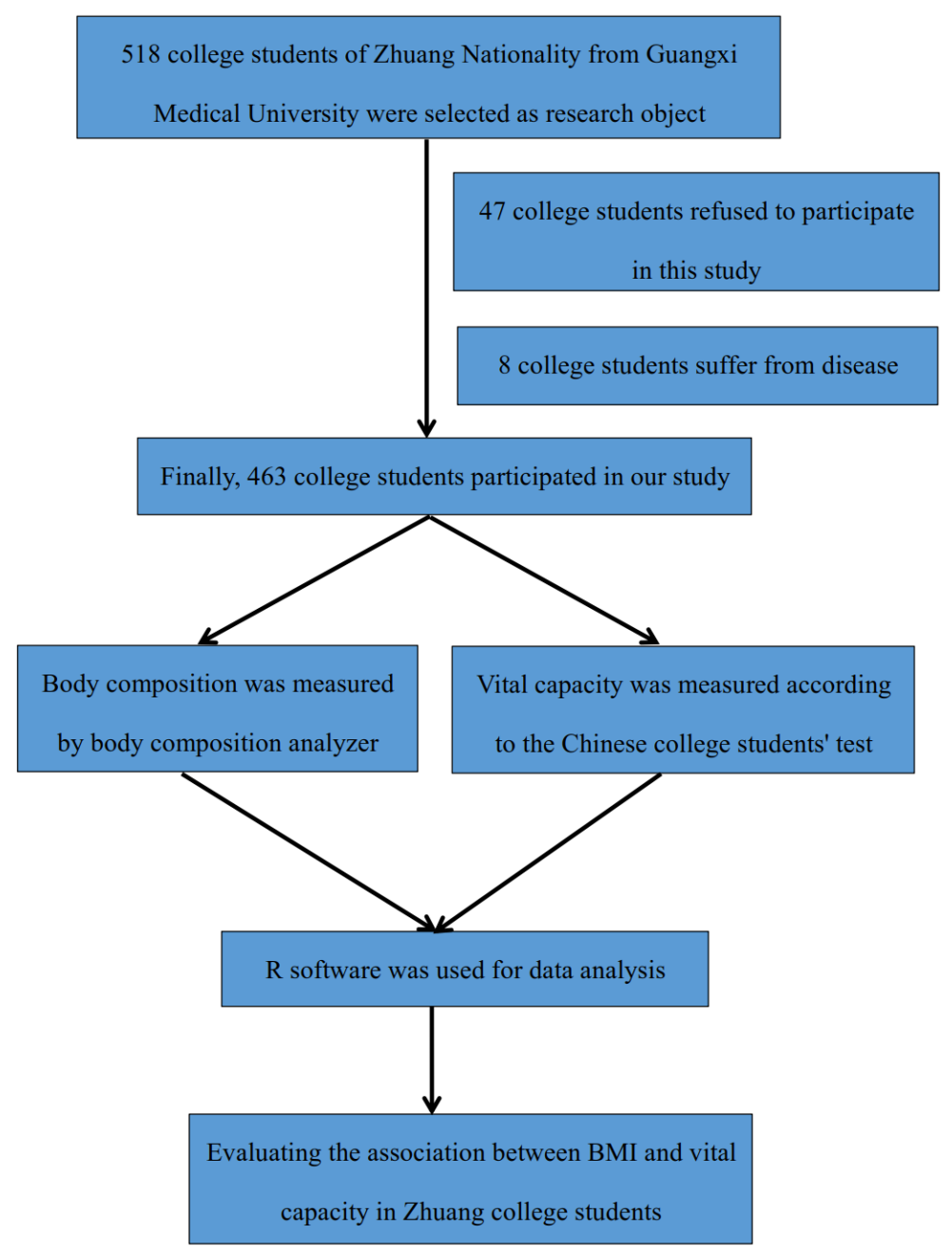

Figure 3: Study population flow chart. 
by altering intercostal muscle function [30]. Chlif M [31] carried out a study recently and pointed out that during exercise, obese volunteers show a decrease in inspiratory muscle activity as a result of reduced inspiratory strength and increased ventilatory drive. Some researchers also pointed out that increased BMI was associated with markers of systemic and vascular inflammation, such as C-reactive protein and leptin [32-34]. The inflammatory factors, such as TNF- $\alpha$, IL-1 $\beta$, IL-6 and TG F- $\beta$, may exert local effects in lung tissue, and lead to subtle reductions in airway diameter.

In our study, the relationship between BMI and VC in college students of Zhuang Nationality is not obvious. With the rise of BMI, VC does not show a downward trend. After adjusting confounding factors by using multivariate regression analysis, the results did not change significantly. Besides, in our analysis stratified by gender, the results also showed no significant change. Our results are inconsistent with previous studies mentioned above. There may be several reasons for this phenomenon. First, the subjects of this study are Chinese, and the difference in population may lead to this phenomenon; second, college students are a healthy group with few diseases. Thus eliminating the influence of diseases (such as hypertension, coronary heart disease, diabetes mellitus), and then further study the independent relationship between BMI and VC. The Zhuang nationality is a special race in Guangxi, China, and few studies have reported this population. Perhaps racial differences can also lead to inconsistent results. Generally, our result is the first study on the association between BMI and VC in college students of Zhuang Nationality. On the other hand, our research also suggests that the mechanisms put forward by former scholars may not apply to college students, and new research mechanisms need to be explored for college students.

\section{Limitations}

A number of limitations should be considered when interpreting findings from this study. First, our analysis was a cross-sectional study, which makes the inference of causality difficult. It remains to be seen whether reducing $\mathrm{BMI}$ will improve $\mathrm{VC}$ in this cohort. Although confounding factors, such as age, body fat, fat and muscle, were controlled by using regression analysis, we cannot completely remove residual confounding due to measurement error. Second, our research subjects are Chinese college students, especially Zhuang college students. Therefore, the findings of our study do not necessarily apply to other populations, such as African college students, European college students and American college students due to environmental factors and dietary factors, etc.

In summary, we found that there was no evidence that $\mathrm{BMI}$ is associated with $\mathrm{VC}$ in college students of
Zhuang Nationality. However, due to small sample size and the presence of potential confounding bias, further studies are required to confirm the conclusion, and to elucidate the mechanisms by which BMI influences VC, particularly in college students coming from different regions and country.

\section{MATERIALS AND METHODS}

518 college students of Zhuang Nationality from Guangxi Medical University were selected as research object. Inclusion criteria: 1) Age $\geq 18$ years; 2) No suffer from disease in the past 1 year, such as tuberculosis, osteoporosis, liver and kidney disease and so on; 3) All college students are aware of this study and written informed consent was obtained from all college students. Exclusion criteria: 1) Non Zhuang college students; 2) Refused to participate in this study; 3) Suffer from a disease in the past 1 year. According to the inclusion criteria and exclusion criteria, 55 college students were excluded from our study (47 college students refused to participate in this study and 8 college students suffer from disease). Flow chart is shown in Figure 3.

\section{Study protocol}

Our research has obtained the approval of the Ethics Committee of Guangxi Medical University, and all patients provided written informed consent. All patients were fully informed about the study protocol and signed informed consent before this study.

\section{BMI}

Height and body weight, were measured using standardized methods to nearest $0.1 \mathrm{~cm}$ or $0.1 \mathrm{~kg}$ in subjects wearing light clothing, and BMI was calculated by dividing body weight $(\mathrm{kg})$ by height squared $\left(\mathrm{m}^{2}\right)$. All college students were divided into five different BMI categories: underweight, $<18.5 \mathrm{~kg} / \mathrm{m}^{2}$; normal BMI, 18.5 $23.9 \mathrm{~kg} / \mathrm{m}^{2}$; overweight, $23.9-27.9 \mathrm{~kg} / \mathrm{m}^{2}$; obese, BMI greater than or equal to $27.9 \mathrm{~kg} / \mathrm{m}^{2}$ (including male college students and female college students).

\section{Body composition}

Body composition, including body fat, fat, muscle, presumption of bone, body moisture, protein, intracellular fluid, extracellular fluid, body fat percentage, muscle volume ratio, visceral fat area, visceral fat, fat content, waist hip ratio, basal metabolism, energy metabolism, swelling index, trunk muscle the amount of the left upper limb muscles, weight; left lower limb muscle mass, right upper limb muscle, right lower limb muscle mass, trunk fat, trunk fat ratio, left upper limb fat, the left upper limb left lower limb fat, fat; left leg fat, right upper limb fat 
Volume, right upper extremity fat percentage and right lower extremity fat mass, were measured by tetrapolar bioelectrical impedance analysis (InBody 3.0, Biospace, Seoul, Korea). All college students wear light clothes when they are measuring, without shoes.

\section{Vital capacity}

The vital capacity (VC) measures the maximum amount of air that can be inhaled or exhaled during a respiratory cycle. It is the sum of the expiratory reserve volume, tidal volume, and inspiratory reserve volume. The inspiratory capacity (IC) is the amount of air that can be inhaled after the end of a normal expiration. It is, therefore, the sum of the tidal volume and inspiratory reserve volume. The total lung capacity (TLC) is a measurement of the total amount of air that the lung can hold. It is the sum of the residual volume, expiratory reserve volume, tidal volume, and inspiratory reserve volume. The unit of $\mathrm{VC}$ is milliliter $(\mathrm{ml})$.

\section{Statistical analyses}

All descriptive data are presented as means and standard deviations or as numbers and percentages. The baseline characteristics for descriptive data were compared using Student's t test, and categorical variables were analyzed using the $X^{2}$ test. We calculated odds ratio (OR) and $95 \%$ confidence intervals $(\mathrm{CI})$ for the associations between body mass index (BMI) and vital capacity.

Non-adjusted models was conduct to adjust for none based on multiple regression analysis; Adjust I model was conduct to adjust for age based on multiple regression analysis; Adjust II model was conduct to adjust for age; body fat; fat; muscle; presumption of bone; body moisture; protein; intracellular fluid; extracellular fluid; body fat percentage; muscle volume ratio; visceral fat area; visceral fat; fat content; waist hip ratio; basal metabolism; energy metabolism; swelling index; trunk muscle the amount of the left upper limb muscles; weight; left lower limb muscle mass; right upper limb muscle; right lower limb muscle mass; trunk fat; trunk fat ratio; left upper limb fat; the left upper limb left lower limb fat; fat; left leg fat; right upper limb fat Volume; right upper extremity fat percentage; right lower extremity fat mass based on multiple regression analysis.

All of the analyses were performed with the statistical software packages R (http://www.R-project.org, The R Foundation). A two-sided significance level of 0.05 was used to evaluate statistical significance.

\section{CONFLICTS OF INTEREST}

The authors declared no conflicts of interest.

\section{FUNDING}

This study was supported by the National Natural Science Foundation of China (NO: 31360259), Guangxi colleges and Universities Key Laboratory of Human Development and Disease Research (NO: 0102402214009C), Key projects of science and technology research in Guangxi Universities (NO: KY2015ZD024) and Guangxi Medical University innovation and entrepreneurship training program (NO: 201610598004, 201610598116, 201710598036 and 201710598030).

\section{REFERENCES}

1. Yach D, Stuckler D, Brownell KD. Epidemiologic and economic consequences of the global epidemics of obesity and diabetes. Nat Med. 2006;12:62-66.

2. Peytremann-Bridevaux I, Santos-Eggimann B. Health correlates of overweight and obesity in adults aged 50 years and over: Results from the survey of health, ageing and retirement in europe (share). Obesity and health in europeans aged $>$ or $=50$ years. Swiss Med Wkly. 2008;138:261-266.

3. Santos R, Aires L, Santos P, Ribeiro JC, Mota J. Prevalence of overweight and obesity in a portuguese sample of adults: Results from the azorean physical activity and health study. Am J Hum Biol. 2008;20:78-85.

4. Leyk D, Rüther T, Wunderlich M, Heiß A, Küchmeister G, Piekarski C, Löllgen H. Sporting activity, prevalence of overweight, and risk factors: Cross-sectional study of more than 12500 participants aged 16 to 25 years. Dtsch Arztebl Int. 2008; 105:793-800.

5. Kim HS, Ham OK, Jang MN, Yun HJ, Park J. Economic differences in risk factors for obesity among overweight and obese children. The J Sch Nurs. 2014;30:281-291.

6. Kelishadi R, Hashemipour M, Sheikhheidar A, Ghatrehsamani S. Changes in serum lipid profile of obese or overweight children and adolescents following a lifestyle modification course. ARYA Atheroscler. 2012; 8:143-48.

7. Bocca G, Corpeleijn E, Stolk RP, Sauer PJ. Results of a multidisciplinary treatment program in 3-year-old to 5-year-old overweight or obese children: A randomized controlled clinical trial. Arch Pediatr Adolesc Med. 2012;166:1109-1115.

8. Wiese MD, Sluggett JK, Wilson CJ, Reuter SE, Turner SC, Evans AM. Perceived and actual paracetamol dosing in overweight and obese children. Eur J Hosp Pharm Sci Pract. 2012;19:438-442.

9. Moges B, Amare B, Fantahun B, Kassu A. High prevalence of overweight, obesity, and hypertension with increased risk to cardiovascular disorders among adults in northwest ethiopia: A cross sectional study. BMC Cardiovasc Disord. 2014;14:155. 
10. Badi MA, Garcia-Triana BE, Suarez-Martinez R. Overweight/obesity and hypertension in schoolchildren aged 6-16 years, Aden Governorate, Yemen, 2009. East Mediterr Health J. 2012; 18:718-22.

11. Wang C, Li J, Xue H, Li Y, Huang J, Mai J, Chen J, Cao J, Wu X, Guo D, Yu L, Gu D. Type 2 diabetes mellitus incidence in Chinese: contributions of overweight and obesity. Diabetes Res Clin Pract. 2015; 107:424-32.

12. Reuter CP, da Silva PT, Renner JD, de Mello ED, Valim AR, Pasa L, da Silva R, Burgos MS. Dyslipidemia is associated with unfit and overweight-obese children and adolescents. Arq Bras Cardiol. 2016; 106:188-93.

13. Pinto Pereira LM, Seemungal TA, Teelucksingh S, Nayak BS. Restrictive pulmonary deficit is associated with inflammation in sub-optimally controlled obese diabetics. J Thorac Dis. 2013; 5:289-97.

14. Van HA, Cabezas MC, Birnie E, van de Geijn GJ, Rudolphus A, Mannaerts G, Njo TL, Hiemstra PS, Braunstahl GJ. Systemic inflammation and lung function impairment in morbidly obese subjects with the metabolic syndrome. J Obes. 2013;2013:131349.

15. Carpio C, Santiago A, García de Lorenzo A, Alvarez-Sala R. [Changes in lung function testing associated with obesity]. [Article in Spanish]. Nutr Hosp. 2014 ; 30:1054-62.

16. Bankhead C, King G. Obesity: Implications for the Clinical Practice of Pulmonary, Critical Care, and Sleep Medicine. MD Conference Express. 2011; 11: 4-5.

17. Saikia K, Barman S, Deka J. Effect of Body Mass Index (BMI) on Forced Vital Capacity (FVC) and Forced Expiratory Volume in first second (FEV1) in young healthy males. Int J Sci Res. 2016; 5.

18. Mohammed Z. Body Fat Percentage(BFP) Versus Body Mass Index(BMI) Which Cassel Parameter Predict Estimated Vital Capacities (VC) and the Maximal Aerobic Capacity(VO2MAX). Cresco Int J Med. 2016; 1.

19. Zhang Y, He M, Wu S, Zhu Y, Wang S, Shima M, Tamura K, Ma L. Short-term effects of fine particulate matter and temperature on lung function among healthy college students in wuhan, china. Int J Environ Res Public Health. 2015;12:7777-7793.

20. Peng R, Li S, Zhang H, Zeng H, Jiang B, Liu Y, Yi X, Xu $\mathrm{M}$, Zhu L, Zhang Z. Weight status is associated with blood pressure, vital capacity, dental decay, and visual acuity among school-age children in chengdu, china. Ann Nutr Metab. 2016; 69:237-45.

21. Dennison EM, Dhanwal DK, Shaheen SO, Azagra R, Reading I, Jameson KA, Sayer AA, Cooper C. Is lung function associated with bone mineral density? Results from the hertfordshire cohort study. Arch Osteoporos. 2013;8:115.

22. Putt MT, Watson M, Seale H, Paratz JD. Muscle stretching technique increases vital capacity and range of motion in patients with chronic obstructive pulmonary disease. Arch Phys Med Rehabil. 2008;89:1103-1107.

23. Jacobs DR Jr, Yatsuya H, Hearst MO, Thyagarajan B, Kalhan R, Rosenberg S, Smith LJ, Barr RG, Duprez DA. Rate of decline of forced vital capacity predicts future arterial hypertension: The coronary artery risk development in young adults study. Hypertension. 2012;59:219-225.

24. Oda E. Low vital capacity was associated with incident diabetes in a japanese health screening population in whom obesity was not prevalent. Can J Diabetes. 2016; 40:143-48.

25. Fukahori $\mathrm{S}$, Matsuse $\mathrm{H}$, Takamura $\mathrm{N}$, Tsuchida $\mathrm{T}$, Kawano T, Fukushima C, Hideaki S, Kohno S. Body mass index correlated with forced expiratory volume in 1 second/forced vital capacity in a population with a relatively low prevalence of obesity. Chin Med J (Engl). 2010;123:2792-2796.

26. Melo LC, Silva MA, Calles AC. Obesity and lung function: A systematic review. Einstein (Sao Paulo). 2014; $12: 120-25$.

27. Steele RM, Finucane FM, Griffin SJ, Wareham NJ, Ekelund U. Obesity is associated with altered lung function independently of physical activity and fitness. Obesity (Silver Spring). 2009; 17:578-84.

28. Manika K, Pitsiou GG, Boutou AK, Tsaoussis V, Chavouzis N, Antoniou M, Fotoulaki M, Stanopoulos I, Kioumis I. The Impact of Pulmonary Arterial Pressure on Exercise Capacity in Mild-to-Moderate Cystic Fibrosis: A Case Control Study. Pulm Med. 2012; 2012: 252345.

29. Paralikar SJ, Kathrotia RG, Pathak NR, Jani MB. Assessment of pulmonary functions in obese adolescent boys. Lung India. 2012; 29:236-40.

30. Poulain M, Doucet M, Major GC, Drapeau V, Sériès F, Boulet LP, Tremblay A, Maltais F. The effect of obesity on chronic respiratory diseases: pathophysiology and therapeutic strategies. CMAJ. 2006; 174:1293-99.

31. Chlif M, Keochkerian D, Feki Y, Vaidie A, Choquet D, Ahmaidi S. Inspiratory muscle activity during incremental exercise in obese men. Int J Obes. 2007;31:1456-1463.

32. Breyer MK, Rutten EP, Spruit MA, Hop WC, Postma DS, Wouters EF. Systemic inflammation in patients with chronic obstructive pulmonary disease: results from the cosmic study. Open J Respir Dis. 2012; 2:63-72.

33. Sin DD, Man SF. Impaired lung function and serum leptin in men and women with normal body weight: A population based study. Thorax. 2003;58:695-698.

34. Aronson D, Roterman I, Yigla M, Kerner A, Avizohar O, Sella R, Bartha P, Levy Y, Markiewicz W. Inverse association between pulmonary function and c-reactive protein in apparently healthy subjects. Am J Respir Crit Care Med. 2006; 174:626-32. 